\title{
Skén\&graphie
}

SKIEN\&GAPHIIU Coulisses des arts du spectacle et des scènes

émergentes

$6 \mid 2019$

Voyages des cultures et des mémoires dans les arts de la scène

\section{Modalités d'appropriation, d'incorporation et de ré-signification des savoirs-danser dans un contexte globalisé - Analyse d'un extrait de Kathak interprété par Sri Pratap Pawar et Akram Khan}

\section{Federica Fratagnoli}

\section{OpenEdition}

Journals

Édition électronique

URL : https://journals.openedition.org/skenegraphie/2898

DOI : 10.4000/skenegraphie.2898

ISSN : 2553-1875

Éditeur

Presses universitaires de Franche-Comté

Édition imprimée

Date de publication : 1 novembre 2019

Pagination : $39-55$

ISBN : 978-2-84867-673-9

ISSN : 1150-594X

\section{Référence électronique}

Federica Fratagnoli, « Modalités d'appropriation, d'incorporation et de ré-signification des savoirsdanser dans un contexte globalisé - Analyse d'un extrait de Kathak interprété par Sri Pratap Pawar et Akram Khan », Skén\&graphie [En ligne], 6 | 2019, mis en ligne le 01 juin 2021, consulté le 27 septembre 2021. URL : http://journals.openedition.org/skenegraphie/2898 ; DOI : https://doi.org/10.4000/ skenegraphie.2898

Ce document a été généré automatiquement le 27 septembre 2021.

Presses universitaires de Franche-Comté 


\title{
Modalités d'appropriation, d'incorporation et de ré- signification des savoirs-danser dans un contexte globalisé - Analyse d'un extrait de Kathak interprété par Sri Pratap Pawar et Akram Khan
}

\author{
Federica Fratagnoli
}

1 «Si mon père [Achhan Maharaj] était encore là aujourd'hui, je me demande s'il reconnaîtrait certaines danses comme du kathak ${ }^{1}$ ». Les mots de Birju Maharaj ${ }^{2}$, l'un des plus éminents représentants d'une pratique artistique originaire du nord de l'Inde, connue sous le nom de kathak, sont très éloquents. Ils parlent de la réorganisation constante et parfois imperceptible que les pratiques artistiques indiennes ont endurée au fil du temps, et qui ont changé inexorablement leurs formes et leurs visées. Émanant d'interventions volontaires ou, parfois, involontaires, ces changements sont le résultat non seulement de la matière mouvante des corps des interprètes qui réactivent les gestes au fil du temps, mais également de la circulation de ces pratiques qui ont voyagé à l'échelle nationale et internationale se confrontant à des contextes sociaux et politiques spécifiques.

2 Si le kathak reste encore couramment associé au domaine du "traditionnel», sa pratique n'a pourtant cessé de se renouveler au cours de l'histoire ${ }^{3}$, et cela au niveau des lieux de représentation, de la mise en scène, du costume et - élément probablement moins ostensible mais non moins décisif - des qualités gestuelles employées pour l'interprétation de ses pièces. L'affirmation des études postcoloniales a ouvert la voie à une nouvelle façon de penser l'histoire des danses de l'Inde, et a remis en cause le récit historiographique conçu dans le cadre du mouvement nationaliste qui a mené l'Inde à l'indépendance du régime colonial britannique. Se faisant porte-voix d'un nouveau 
discours, l'historiographie postcoloniale a permis ainsi de mettre en lumière la « modernité » des pratiques indiennes, et de dévoiler certaines réalités historiques liées à la colonisation anglaise et omises pendant les années précédentes. Elle a notamment montré que le kathak - comme d'autres styles de danses «traditionnelles » indiennes -, n'était pas le reflet d'une tradition ancienne et immuable, mais plutôt une pratique « en mouvement ", qui avait été d'ailleurs reconstruite au cours des années 1930 et 1940, à partir d'un processus d'institutionalisation, gentrification et partielle sanskritisation ${ }^{4}$.

C'est sous cette nouvelle forme que ce style de danse de l'Inde, décrété «classique » au cours des années $1950^{5}$, s'est progressivement affirmé au niveau international, notamment en concomitance avec le mouvement de la diaspora indienne. L'apprentissage et la pratique des danses «classiques" indiennes en dehors du subcontinent - tels que le kathak -, ont joué un rôle important sur le plan identitaire, car elles ont permis aux immigrants indiens de garder un lien avec leurs racines, malgré l'expérience du déplacement. Pour les nouvelles générations, ces pratiques ont permis avant tout d'intégrer les traditions culturelles indiennes et les enseignements moraux et religieux ${ }^{6}$, préservant une attache avec un contexte lointain et parfois étranger.

De ce fait, la pratique du kathak a enduré des réajustements et des variations esthétiques plus ou moins importants en fonction de ces nouveaux lieux d'accueil, et des orientations esthétiques des acteurs qui la pratiquent. «L'essence du voyage [...] est non seulement rencontre de l'autre, mais plus encore transformation de soi au moyen de cette rencontre ${ }^{7}$, précise Venayre Sylvain dans un article sur l'histoire culturelle du voyage. Ainsi, la circulation de la technique du kathak a induit non seulement la rencontre et la confrontation avec d'autres techniques de danse, mais aussi et surtout une transformation de la technique elle-même.

5 Les pages de cet article s'intéresseront à la pratique «traditionnelle » du kathak et à sa circulation dans le contexte anglais; elles tenteront d'interroger les variations parfois imperceptibles que son esthétique a enduré en dehors du subcontinent indien. Alors que la circulation des pratiques artistiques est souvent pensée au niveau des "territoires géographiques", nous avons fait ici le choix de transposer cette notion à l'échelle du sensible et d'aborder la circulation au niveau des «territoires corporels ». Ce changement de perspective permettra d'étudier la manière dont la technique du kathak "voyage " dans et à travers la corporéité des interprètes, impulsée par des processus de passation et d'incorporation.

6 Nous nous appuierons sur une étude de cas singulier, portant sur deux interprètes reliés par une filiation artistique : le maître Sri Pratap Pawar et son disciple Akram Khan. Ces deux artistes sont aujourd'hui considérés comme des figures emblématiques du kathak dans le panorama de la danse indienne anglo-saxonne. L'analyse d'un bref extrait de kathak «traditionnel » exécuté par ces deux artistes constituera l'objet de notre étude. L'extrait en question, d'une durée de 35 secondes, apparaît dans une vidéo documentaire sur Akram Khan, qui a pour titre «South bank show: Akram Khan ${ }^{8}$; elle date de 2002. Cette courte vidéo revêt un intérêt particulier car les deux artistes dansent ensemble le même enchaînement chorégraphique ${ }^{9}$. Cette circonstance, assez rare, permettra une lecture comparative du geste et de son fond tonique et permettra de repérer l'écart qualitatif propre à la façon de se mouvoir et d'investir l'espace des deux interprètes. 


\section{Les artistes : Sri Pratap Pawar et Akram Khan}

7 Cette présentation n'a pas l'ambition d'être exhaustive. Elle se propose de retracer quelques moments saillants de leur carrière, apportant ainsi des indications sur leur formation et leurs choix artistiques.

8 Sri Pratap Pawar ${ }^{10}$ a contribué de manière importante au développement et à l'affirmation de la danse indienne en Angleterre. Né à Dhar, Madhya Pradesh (Inde), en 1942, il a été formé au kathak en Inde, notamment à l'école Kathak Kendra de Delhi. Il est considéré comme l'un des premiers disciples du gourou Birju Maharaj, le plus important représentant de l'école (gharana) Lucknow ${ }^{11}$. Après une remarquable carrière en Inde, dans les années 1970, il voyage à l'international comme ambassadeur culturel avec son épouse Prya Pawar, danseuse d'odissi et de kathak, pour « rendre populaires les formes de danse indienne à l'étranger $»^{12}$. Après trois années passées en Guyana, il est déplacé à Trinidad, où il poursuit l'enseignement du kathak et crée plusieurs spectacles $^{13}$. C'est seulement à partir des années 1980 que Sri Pratap Pawar et son épouse s'installent en Angleterre, où ils fondent la compagnie de danse " Triveni Dance Company ", qui a effectué des tournées en Asie, Europe, Amérique, ainsi qu'en Afrique et dans les Caraïbes.

Bien que sa pratique et son enseignement soient toujours restés fidèles à la " tradition » du style Lucknow, à partir de la moitié des années 1970 ses spectacles tentent de faire dialoguer le kathak avec d'autres langages corporels, dont le flamenco et les danses afro-caribéennes. Pour nommer ces types de spectacle, qui revêtent un intérêt particulier pour notre étude, il utilise le terme de jugalbandi, littéralement : « jumeaux entrelacés ». Propre au champ musical, ce terme indique une performance de musique hindustani ${ }^{14}$ - ou dans certains cas carnatique - à laquelle participent deux musiciens, considérés tous les deux comme solistes. Pratap Pawar déplace ce terme - et le concept qu'il véhicule - dans le domaine de la danse. Il l'utilise ainsi pour désigner ses spectacles, conçus sous forme de "duo ", qui mettent en scène principalement la rencontre entre le kathak et le flamenco ou les danses afro-caribéennes.

Remarquons que dans ce type de spectacle, la pratique «traditionnelle » est réinvestie au sein d'un dispositif spectaculaire où les deux langages chorégraphiques coexistent de façon autonome sur scène, l'un à côté de l'autre. Il convient de noter que dans ces créations chacun des artistes reste maitre de sa pratique et l'interaction, qui prend souvent la forme d'une compétition ludique, ne déstabilise nullement le style et la qualité de la danse et, par conséquent, le système perceptif des interprètes. Ce format sera interrogé et remis en discussion par la nouvelle génération d'artistes indiens, qui envisageront bien d'autres manières de penser le dialogue interculturel sur scène.

11 Disciple de Sri Pratap Pawar, Akram Khan ${ }^{15}$ a commencé l'apprentissage du kathak à l'âge de sept ans à la National Academy of Indian Dance (NAID) de Londres ${ }^{16}$, sous la tutelle de son futur maître. Né à Londres en 1974, d'une famille originaire du Bangladesh installée en Angleterre à la fin des années 1960, Akram Khan reçoit une formation qui se place à l'intersection entre deux cultures, «l'éducation britannique, dans la sphère publique, et la culture bengali, dans la sphère privée ${ }^{17}$. Le même Akram Khan décrit, lors d'un entretien, cette double appartenance : "Encore maintenant je pense à notre porte comme à une espèce de Stargate: en dehors d'elle je suis un asiatique né en Angleterre, mais quand j'en passe le seuil, je deviens un asiatique de l'Inde ${ }^{18}$.» À l'âge de trois ans, il danse déjà lors de fêtes familiales les danses 
folkloriques du Bangladesh, tout en étant simultanément fasciné par les chorégraphies et les vidéo-clips de Michael Jackson. Vers la moitié des années 1980, il est engagé dans son premier rôle professionnel, et joue, entre 1985 et 1989, le jeune fils dans la production théatrale du Mahabharata de Peter Brook, et le personnage d'Ekalavya dans le film homonyme. Souhaitant faire de la danse sa carrière, au début des années 1990, il ratifie son statut de disciple du maître Sri Pratap Pawar à travers une cérémoniespectacle, qui scelle son engagement et sa filiation artistique. Encouragé par ses parents, Akram Khan entame successivement des études universitaires choisissant un diplôme en danse contemporaine. Il s'inscrit initialement à la De Montford University à Lester et puis à la Northern School of Contemporary Dance à Leeds, où il obtient son diplôme. C'est au cours de ces années de formation, qu'Akram Khan découvre certains styles de danse contemporaine occidentale ${ }^{19}$, et expérimente de nouvelles manières d'utiliser son corps et de penser le mouvement. Sa double formation, tout à fait inhabituelle pour un danseur de kathak des années 1990, contribue à rendre complexe et ambigu son statut d'interprète, placé entre deux communautés artistiques et donc " entre » deux formes d'expression identitaire. En 2000, grâce à l'obtention d'une bourse d'étude, Akram Khan participe au projet «X-Group " ${ }^{20}$, à PARTS ${ }^{21}$, une résidence pour jeunes chorégraphes consacrée à la conception de nouveaux langages chorégraphiques. C'est au cours de cette période qu'il élabore sa propre esthétique, qui s'alignera sur les valeurs de la danse contemporaine européenne, avec une attention particulière aux questions politiques et sociales. Sa posture d'insider/outsider ${ }^{22}$, à cheval entre deux cultures et deux manières de faire et concevoir le mouvement, finira par devenir, avec le temps, son indiscutable point fort, décidant de la complexité de son langage chorégraphique et de ses choix en matière esthétique.

Se superposant à sa pratique du kathak, les principes de la danse contemporaine occidentale ont induit une véritable réorganisation de ses schémas sensori-moteurs et de ses coordinations, et ont influencé non seulement sa carrière de danseur et de chorégraphe " contemporain ", à travers la création d'un nouveau langage qui prendra le nom de "contemporary kathak », mais aussi sa manière de danser et d'interpréter les pièces de kathak «traditionnel». Laissant volontairement de côté ses créations « contemporaines » qui l'ont rendu fameux au niveau international, cet article propose d'étudier les spécificités de son kathak «traditionnel $»^{23}$. Bien que fidèle aux formes et aux codes transmis par le maître Sri Pratap Pawar, ses interprétations se caractérisent par une qualité singulière, qui, comme nous le verrons, répond à une réorganisation profonde et involontaire de ses patterns sensori-moteurs, informés par l'esthétique de la danse contemporaine européenne.

\section{Analyse d'un extrait de kathak « traditionnel » ${ }^{24}$}

https://www.youtube.com/watch ?v =nOiGl-JyTVw

(Analyse de la section de 9'15" à 9'50")

13 Cette section de l'article sera consacrée à l'analyse de l'extrait de danse kathak exécuté par Akram Khan et son maître Sri Pratap Pawar. Comme précisé dans l'introduction, l'intérêt de cet extrait réside dans la proximité des deux artistes. Alors qu'il est difficile de définir une qualité gestuelle en soi, indépendamment d'un point de repère extérieur ${ }^{25}$, la présence des deux interprètes permet une observation fine des écarts, la corporéité de l'un constituant une base de comparaison concrète pour la lecture du 
geste de l'autre. Les outils méthodologiques mobilisés pour l'analyse procèdent essentiellement de l'Analyse Fonctionnelle du Corps dans le Mouvement Dansé26 et de la théorie de l'Effort et de l'Effort Shape de Rudolf Laban ${ }^{27}$. Se limitant à la lecture de certains aspects choisis du mouvement - l'attitude envers la gravité, voire la façon d'organiser le "pré-mouvement ${ }^{28}$, et la manière d'investir l'espace environnant -, notre analyse se veut tout simplement « un trajet possible du regard $»^{29}$ parmi d'autres. Faute d'exhaustivité, elle aura cependant le mérite de défendre un angle d'approche singulier et de tenter la mise en mot de deux manières spécifiques d'exécuter - et donc d'interpréter - un même geste.

L'attitude des deux interprètes envers la gravité constitue notre premier point d'intérêt. Il faut remarquer qu'au cours de l'enchaînement chorégraphique, les deux artistes n'abandonnent jamais leur posture verticale ${ }^{30}$. Érigés sur leurs appuis, leurs mouvements s'articulent autour d'un axe central, engendré par ce qu'Hubert Godard appelle la première articulation fondatrice ${ }^{31}$ qui différencie un haut d'un bas ${ }^{32}$. Construite à partir de deux sens du poids séparés - un qui s'organise à partir des appuis au sol (poids d'en bas), et un qui s'organise à partir du vestibule et des yeux (poids d'en haut) -, la verticalité des deux interprètes se distingue par une organisation spécifique de ces deux polarités. Dans l'interprétation de Sri Pratap Pawar, le haut de la cage thoracique semble revêtir une importance particulière lors de l'exécution du mouvement. Le maitre semble littéralement " porté » par cette partie du corps, qui est souvent dans une légère extension et qui tient et dirige le mouvement. Sa construction gravitaire, qui privilégie donc le haut - non sans rappeler l'organisation corporelle d'un danseur de claquettes - donne au spectateur une sensation de suspension, qui libère et allège les membres inférieurs du corps. Akram Khan présente en revanche une organisation gravitaire plus homogène, le curseur entre les deux polarités étant moins orienté. Un lien bien actif avec le haut - repérable par l'activation du vestibule et l'extension au niveau du cou et de la tête - est contrebalancé par un ancrage assez important au sol, rendu manifeste par un lâcher-prise du poids du corps, surtout au niveau du sacrum. La tactilité très prononcée des plantes des pieds et le lien direct ischions-talons renforcent cette sensation d'ancrage. La clarté d'orientation entre les deux polarités tend à créer un espace homogène et à libérer la partie centrale du corps de tensions superflues, laissant ouvert un maximum de possibilité de mouvement.

Lors de l'exécution de l'enchaînement, les deux interprètes mettent en place des stratégies d'organisation kinesthésiques singulières, qui induisent une différente mobilisation des parties du corps.

La construction gravitaire de Sri Pratap Pawar tend à stabiliser le haut de sa cage thoracique, la rendant ainsi moins mobile, notamment au niveau des micro-mobilités intrinsèques à cette partie du corps. Cela est visible surtout lors des mouvements circulaires du bras, la tête accompagnant avec une extension vers l'arrière. Alors qu'Akram Khan a un haut de cage bien articulé - ce qui lui permet un mouvement d'épaulé et une légère torsion qui entraîne la colonne et les épaules légèrement en spirale -, son maître mobilise le buste essentiellement en inclinaison, privilégiant ainsi le plan frontal.

17 En outre, Sri Pratap Pawar exécute les gestes à partir d'un moindre déplacement de ses membres supérieurs et inférieurs ainsi que de son buste. Ce sont d'avantage les avantbras (du coude à la main) et les bas de jambes (du genou au pied), qui sont pris par le mouvement. Le reste du corps - le buste, avec les cuisses et les bras - a tendance à 
former une structure compacte qui assure une stabilité. Cela implique qu'il y a peu d'articulations au niveau de la ceinture scapulaire et de la ceinture pelvienne et que le mouvement se fait principalement dans la partie périphérique du corps. Au niveau qualitatif, ce type d'organisation kinesthésique donne à voir des mouvements très précis et efficaces, qui, pour utiliser les mots de Laban, se réalisent à partir d'un espace " direct » et d'un temps "soudain ». L'efficacité et la précision, voire la concision, du geste priment. Cette organisation permet d'ailleurs à Sri Pratap Pawar de gagner en vitesse. Akram Khan en revanche présente une mobilisation plus importante de la partie centrale du corps. La disponibilité et la mobilité de la cage thoracique et des articulations au niveau de la ceinture scapulaire et de la ceinture pelvienne assurent la transmission du mouvement entre axe et membres, laissant ressortir clairement les liens physiologiques entre ces parties du corps. De manière plus générale, Akram Khan donne à voir une corporéité compacte mais "disponible", car dénuée de tensions musculaires superflues, ce qui permet au mouvement de circuler plus librement entre les diverses parties du corps. La sensation d'un mouvement qui se fait en accord avec la gravité et la respiration et qui exploite le momentum (impulsion), renvoie l'observateur à une esthétique bien présente dans certains styles de danse contemporaine, dont la release technique ou le contact improvisation. Remarquons également que la mobilisation très prononcée du buste, qui exalte le volume du corps, induit l'apparition d'un espace «tridimensionnel ». Révélant une conscience accentuée de la distance qui se joue entre le devant et le derrière (la deuxième articulation fondatrice), son mouvement s'inscrit dans un jeu de porosité et d'échange, qui laisse ressortir les volumes et l'épaisseur du corps. Si cette conscience lui permet de gagner en prise d'espace, elle induit en revanche une subtile perte en concision de mouvement, basculant vers une utilisation de l'espace « indirecte».

18 La conscience volumétrique du geste d'Akram Khan influence également l'exécution des pirouettes (chakkars), élément technique important dans le kathak. Alors que Pratap Pawar tourne autour d'un centre clairement défini - un point pivot qui se situe au niveau de la cage thoracique, en correspondance de la septième/huitième vertèbre dorsale -, Akram Khan organise sa pirouette en fonction du volume de son corps, ce qui instaure un autre type de temporalité et un autre pré-mouvement lors de l'exécution des pirouettes. L'épaisseur du volume amplifie son mouvement et induit un imperceptible étirement du temps, par rapport à l'exécution de Sri Pratap Pawar.

19 L'utilisation du regard est également intéressante pour notre étude, car elle témoigne de la manière de s'inscrire dans l'espace et d'y projeter son mouvement. Les yeux de Sri Pratap Pawar ont tendance à suivre le mouvement de ses mains de très près. Il semble utiliser son regard principalement en mode « focal » ou "directionnel " ${ }^{33}$, pointant et désignant l'espace de façon nette et extrêmement concrète. Akram Khan en revanche semble prendre de la distance par rapport aux éléments observés, notamment ses mains. Son regard est plus abstrait que celui de Sri Pratap Pawar et, tout en restant en modalité « focale ", il ouvre au "périphérique " ${ }^{34}$. La différente utilisation du regard est très claire au moment de la pose finale. Le regard de Sri Pratap Pawar est présent et direct, alors que le regard d'Akram Khan se projette loin dans la kinesphère, et traverse l'espace s'inscrivant dans une profondeur. La position de la tête et du sternum, légèrement soulevés, en sont des manifestations significatives.

20 La manière dont les gestes des deux interprètes s'inscrivent dans l'espace environnant s'articule donc de façon différente. Pour tenter de nommer cette différence, nous 
convoquerons la théorie de l'Effort Shape de Rudolf Laban, et plus précisément les "Modes de Changement de la Forme" (Modes of Shape Change), système qui vise à décrypter la transformation du corps dans l'échange qu'il instaure avec l'espace environnant. En effet, comme Rudolf Laban le rappelle dans ses écrits, «l'espace est le trait caché du mouvement et le mouvement un aspect visible de l'espace ${ }^{35}$. Dans ce sens, l'espace d'inscription du mouvement - et surtout la relation que le danseur entretient avec celui-ci - devient le point de référence privilégié de l'attitude corporelle, ou bien de la «shape $»^{36}$ du danseur.

21 L'écart existant entre un mode directionnel (ou « directional ») et un mode plastique (ou "carving»), semble rendre compte de la manière dont les corporéités des deux interprètes interagissent avec l'espace. L'interprétation de Sri Pratap Pawar répond davantage à un mode "directionnel », caractérisé par la notion de direction et de " ciblage ». Ce mode s'intéresse à la visée d'un geste et donc à la trajectoire tracée par une partie du corps afin de rejoindre un point précis dans l'espace. Fonctionnant par rapport à des lignes droites, il est en lien avec une utilisation du corps qui se déploie de manière bi-dimentionnelle, modalité qui a été d'ailleurs associée par d'éminents critiques au style kathak ${ }^{37}$. L'interprétation d'Akram Khan répond en revanche au mode " plastique ", car sa corporéité semble épouser l'espace environnant et faire ressortir le volume. Le mode plastique fait effectivement appel à une utilisation volumétrique du corps et donc à une pleine mobilité de ses membres, notamment au niveau des articulations des hanches et de la cage thoracique. Or, cette manière d'interagir avec l'espace environnant influence les interprétations «traditionnelles » d'Akram Khan. La bi-dimensionnalité du kathak de Sri Pratap Pawar est bouleversée par une réimposition du volume propre à la corporéité de l'interprète, qui finit, de cette façon, par épouser l'espace environnant. Alors que les pas exécutés restent analogues et conformes aux figures prescrites par la «tradition », l'interprétation d'Akram Khan réinvente la façon de s'inscrire dans l'espace et de l'investir, ainsi que la façon de jouer avec la gravité et de gérer les transferts du poids ${ }^{38}$.

22 Au cours de cette analyse, nous avons essayé de démontrer à quel point la notion de «tradition» est matière mouvante, sans cesse déplacée par les circulations des pratiques artistiques et par les apports singuliers des acteurs qui s'en emparent. L'étude présentée a permis de pointer du doigt une réalité singulière. Elle a montré de quelle manière l'interprétation d'un même enchaînement de kathak peut diverger sur le plan qualitatif, et induire ainsi l'apparition d'une esthétique singulière. Notre hypothèse est que l'incorporation de plusieurs techniques de danse contemporaine ${ }^{39} \mathrm{a}$ réorganisé qualitativement les schémas sensori-moteurs d'Akram Khan, agissant involontairement sur l'exécution de son geste ${ }^{40}$. Tout en poursuivant la filiation de son maître, le kathak de cet artiste semble répondre désormais à des principes kinesthésiques autres, témoignant d'un lien étroit avec le contexte londonien et les pratiques artistiques occidentales.

Akram Khan propose ainsi quelque chose de fortement novateur. Résultat d'un long processus de négociation et d'élaboration proprioceptive, l'interaction des cultures n'est pas, pour Akram Khan, un exercice intellectuel, mais une réalité physique. Incarnant plusieurs positions identitaires ainsi que plusieurs vocabulaires de mouvement - ce que Guy Cools appelle une "identité somatique »"11 -, la corporéité d'Akram Khan a ainsi négocié, en première personne, les tensions et les potentialités d'une société multiculturelle et globale. Reprenant la pensée de Ramsey Burt ${ }^{42}$, cette 
négociation est importante car elle a le potentiel de suggérer de nouvelles possibilités de coexistence égalitaire. En ce sens, le travail proposé par Akram Khan semble ouvrir un espace de négociation, qui est aussi et surtout un espace d'ouverture à la différence et à l'altérité.

\section{NOTES}

1. Leea \& Pasrcha venkataraman, Avnash, La danse classique indienne. Une tradition en transformation, Paris, EDL, 2003, p. 44.

2. Birju Maharaj (1938) descend d'une famille d'artistes. Son père Achhan Maharaj, ainsi que son oncle Bindadin Maharaj ont en effet dansé pour les derniers patrons royaux de kathak, au cours des dernières décennies du XIX ${ }^{\mathrm{e}}$ siècle. Encore aujourd'hui Birju Maharaj est considéré comme l'un des plus remarquables représentants de l'école (gharana) Lucknow. (Ramsay BURT, "Contemporary dance and the performance of multicultural identities", Conference on research in dance, Copenhagen, The Ministry of Culture Committee on Sports Research, 8-9 janvier 2004, p. 5).

3. L'histoire du kathak reste controversée et divergente. Un certain nombre de chercheurs s'accordent cependant sur l'idée d'une forme artistique née d'un syncrétisme culturel et formel. Voir entre autres : Margaret WALKER, Kathak Dance. A Critical History, Phd, University of Toronto, 2004, et Chakravorty PALLABI, Belles of Change. Kathak Dance, Women and Modernity in India, Calcutta, Seagull Books, 2008.

4. Margaret WALKER, «Revival and Reinvention in India's Kathak Dance », MUSICultures 37, 2010, p. 176.

5. Purnima SHAH «State Patronage in India: Appropriation of the "Regional" and "National" ", Dance Chronicle, $\mathrm{n}^{\circ}$ 1, vol. XXV, 2002, p. 125-141 et Urmimala SARKAR MUNSI, «Boundaries and Beyond: Problems of Nomenclature in Indian Dance History ", dans Urmimala SARKAR MUNSI, (dir.), Dance: Transcending Borders, New Delhi, Tulika Books, 2008, p. 78-98.

6. Ann R. DAVID, «Local Diasporas/Global Trajectories New Aspects of Religious 'Performance' in British Tamil Hindu Practice ", Performance Research, 2008, Vol. 13, Issue 3, p. $89-99$ et «Religious Dogma or Political Agenda? Bharatanatyam and its Reemergence in British Tamil Temples», Journal for the Anthropological Study of Human Movement, Vol. $14 \mathrm{n}^{\circ}$ 4, 2007.

7. Venayre sYLVAIN, "Présentation: Pour une histoire culturelle du voyage au XIX ${ }^{\mathrm{e}}$ siècle", Sociétés \& Représentations, 2006/1, n²1, p. 5.

8. "The South Bank Show ", Akram Khan. Vidéo documentaire en trois parties sur le parcours artistique d'Akram Khan, passée sur les chaînes anglaises en 2002. Analyse de la section de 9'15" à 9'50". https://www.youtube.com/watch?v= nOiGl-JyTVw, consulté le 2 juin 2018.

9. Dans la vidéo, l'enchaînement chorégraphique est présenté de la manière suivante : «Baran, composition of percussive syllables executed with vigour ».

10. Pour une analyse plus approfondie de la biographie de Sri Pratap Pawar nous renvoyons le lecteur à l'ouvrage d'Ashish MOHAN KHOKAR, My Journey In Dance: Guru Pratap Pawar (Clarion Books, New York, 2011), ainsi qu'à l'article « Pratap Pawar: Global Guru of Kathak », Nartaki (en ligne), http://www.narthaki.com/ info/tdhc/tdhc24.html, consulté le 28 mai 2018.

11. Il existe deux grandes écoles (gharana) de kathak, dont les noms sont issus des villes dans lesquelles elles se sont développées : le gharana de Jaipur et le gharana de Lucknow. Ces styles se 
distinguent par l'importance prépondérante donnée à l'aspect rythmique, dans le premier, et à la grâce et à l'exactitude des mouvements, dans le second. Même si cette différenciation est encore actuelle, la distinction entre gharana, est en train de devenir de plus en plus floue et hors de propos, pour être simplement porteuse du désir de préserver des «identités » et des " origines » distinctes (Pasricha VenKATARAMAn, op. cit.).

12. «to popularise Indian dance forms abroad ». Citation présente sur : http://pratappawar.co.uk/ about/biography.html, consulté le 28 mai 2018.

13. Ashish монаN кHоKAR, «Pratap Pawar: Global Guru of Kathak», Nartaki (en ligne), http:// www.narthaki.com/info/tdhc/tdhc24.html, consulté le 28 mai 2018.

14. L'expression "musique hindustani » désigne la tradition musicale du nord du subcontinent indien, qui diverge du système propre au sud, qui est connu sous le terme de «musique carnatique $»$.

15. Pour une analyse plus approfondie de la biographie de Akram Khan nous renvoyons le lecteur à Royon MITRA, Akram Khan: Dancing New Interculturalism, Palgrave Macmillan, New York, 2015, Guy Cools, In between dance cultures. On the migratory artistic identity of Sidi Larbi Cherkaoui and Akram Khan, Valiz, Amsterdam, 2015, Annalisa PICCIRILLo, « Hybrid Bodies in Transit: the 'third language' of contemporary kathak», Anglistica, Napoli, Università degli Studi di Napoli "L'Orientale", Vol. 12, nº 2, 2008, p. 27-41 (disponible sur: http://www.anglistica.unior.it/sites/ anglistica/files/04\%20 Piccirillo.pdf. Consulté le 20 mars 2014), Ramsey Burt, «Contemporary dance and the performance of multicultural identities", Conference on research in dance, The Ministry of Culture Committee on Sports Research, Copenhagen, 8-9 janvier 2004, p. 1-13 (disponible sur: http://www.akramkhancompany.net/wp-content/uploads/ 2016/01/kaashessay.pdf. Consulté le 3 mai 2018).

16. Royona MITRA, op. cit., p. 7.

17. Ibid., p. 34.

18. Dans « Massive Kathak», The Sunday times, 6 avril 2003. «Even now I think of our front door as a kind of Stargate: outside I'm a British-born Asian, but when I step through it, I become Asian from India ».

19. Dans un entretien avec Patrick Bensard, in Xavier Fischer, Dancer's Studio: Akram KHAN, DVD, Vol. 1, Injam Production, Monaco Dance Forum, Mezzo, 2003, Akram Khan affirme avoir étudié, pendant sa formation en danse contemporaine, la technique Graham, Release, Cunningham, Alexander et le Physical theatre. Royona Mitra, dans Akram Khan: Dancing New Interculturalism, op. cit., précise qu'il a également étudié la danse classique et le contact improvisation.

20. Royona MITRA, op. cit., p. 45 et Guy Cools, In between dance cultures. On the migratory artistic identity of Sidi Larbi Cherkaoui and Akram Khan, Valiz, Amsterdam, 2015 p. 45.

21. Fondé en 1994 par la chorégraphe Anne Teresa De Keersmaeker et Bernard Foccroule, directeur de l'opéra nationale La Monnaie, PARTS (Performing Arts Research and Training Studio) est une école de formation en danse contemporaine, fondée sur la pratique artistique d'Anne Teresa De Keersmaeker et d'autres chorégraphes.

22. Royona MITRA, op. cit., p. 23.

23. À côté d'une production, conçue dans une perspective plus proprement "contemporaine », Akram Khan a continué à présenter en soliste ses programmes de kathak «traditionnels »: Polaroid Feet (2000), Ronin (2003), Gnosis (2010).

24. Je tiens à remercier Odile Roquet et Emmanuelle Lyon, qui ont gentiment partagé avec moi leurs regards experts, ainsi que les étudiants du master «Études en danse » de l'Université de Nice Sophia Antipolis, qui ont apporté leur contribution à la lecture de cet extrait vidéo.

25. Toute lecture qualitative du geste est en premier lieu une "pensée du relatif ", dépendant toujours d'un point de comparaison, soit-il explicite ou implicite. L'interprétation d'une qualité ou d'un effort sera toujours dépendante de la situation de départ - le « point d'où l'on vient »-, 
ou d'une situation idéale, voire de repères internes et imaginaires, qui constituent la base de comparaison.

26. Constituée en France autour des années 1990, l'Analyse Fonctionnelle du Corps dans le Mouvement Dansé (AFCMD), plus communément appelée Analyse du Mouvement, est une "discipline favorisant la compréhension et l'intégration des mouvements en danse " (Philippe LE MOAL (dir.), Le Dictionnaire de la danse, Paris, Larousse, 1999, p. 676). Elle « interroge l'intention $\mathrm{du}$ geste et l'organisation posturale de la personne, dans un contexte d'action défini, en instaurant un dialogue ouvert avec l'imaginaire du mouvement " (site de l'AFCMD, Association accord cinétique : http://afcmd.com/page/11/qui-sommes-nous, consulté le 2 juin 2018).

27. La théorie de l'effort, développée par Rudolf Laban au cours des années 1940, envisage une analyse qualitative du mouvement à partir des quatre facteurs du mouvement: espace, temps, poids, flux. Dans les années 1950, en étroite collaboration avec l'anglais Warren Lamb, Rudolf Laban élaborera un autre concept d'analyse qualitative du mouvement, appelé "shape ", qui offrira une description du mode de transformation du corps suivant principalement son rapport à l'espace.

28. Le pré-mouvement est une « attitude envers le poids, la gravité, qui existe déjà avant que nous bougions, dans le seul fait d'être debout, et qui va produire la charge expressive du mouvement que nous allons exécuter. [...] C'est lui qui détermine l'état de tension du corps et qui définit la qualité, la couleur spécifique de chaque geste». Hubert Godard, "Le geste et sa perception », dans Isabelle GINOT, Marcelle MICHEL, La Danse au XXe siècle, Paris, Larousse, 2002 [1995], p. 236.

29. Julie PERRIN, Figures de l'attention. Cinq essais sur la spatialité en danse, Paris, Les presses du Réel, 2012, p. 19.

30. Erwin STRAUS, «La posture érigée ", Quant à la danse, $n^{\circ} 1$, Octobre 2004, Images En Manœuvres Éditions/Le Mas de la Danse, p. 22-42.

31. Hubert Godard distingue trois articulations fondatrices : l'articulation haut-bas, qui permet la constitution de l'axe, l'articulation devant-derrière, qui régit le processus de construction de l'adossement, l'articulation gauche-droite, qui permet la latéralisation et l'apparition de la torsion - qui assure l'élasticité de la distance avec l'autre. À ces trois articulations fondatrices, il en rajoute une quatrième, relative au champ de présence, qu'il appelle "vergence ». (Mathieu BOUVIER et Loïc TOUZE, Fond / figure. Entretien avec Hubert Godard, entretien réalisé aux laboratoires d'Aubervilliers le 7 novembre 2013. Consulté sur la plate-forme numérique «Pour un atlas des figures » conçue par Mathieu Bouvier et Loïc Touzé le 10 mars 2017).

32. Mathieu BOUVIER et Loïc TOUZE, op. cit.

33. Isabelle GINOT, « Regarder », dans Marie GLON \& Isabelle LAUNAY (dir.), Histoires de gestes, Paris, Actes sud, 2012, p. 220-221.

34. Emmanuelle Lyon parle à ce propos de regard « orienté ».

35. Rudolf LABAN, Espace dynamique. Choreutique, Bruxelles, Nouvelles de danse, 2003, p. 76.

36. Le choix du terme shape - qui s'oppose au terme anglais forme, utilisé plutôt dans le sens de « contour » ou «silhouette» - met en relief l'idée que la transformation de la forme est conçue principalement à partir d'une vision intérieure de la corporéité, qui reste le point de référence privilégié de toutes interprétations qualitatives.

37. Kapila vatsyayan, Indian classical dance, New Delhi, Publications Division, 1997, p. 88. «Kathak, like miniature painting is two-dimensional in character. It conceives of space only in straight lines and does not attempt to give a three-dimensional effect so characteristic of the relief in indian sculpture ».

38. Federica FRATAGNOLI, "Le langage chorégraphique d'Akram Khan. Eléments de style d'un kathak 'contemporain' ", in Federica Fratagn FRATAGnoli oli, Mahalia Lassibille (dir.), Danser contemporain. Gestes croisés d'Afrique et d'Asie du Sud, Montpellier, Deuxième Époque, p. 109-136. 
39. Probablement, il serait restrictif d'attribuer exclusivement à l'étude des techniques de danse occidentale - et donc à sa seule formation de danseur - le fait que sa corporéité s'exprime au travers de canons novateurs. Le simple fait d'avoir grandi et vécu dans le contexte de la contemporanéité londonienne lui a certainement imposé une attitude corporelle spécifique.

40. Akram Khan affirme à plusieurs reprises que son langage chorégraphique est le fruit d'un processus involontaire : «It was not a conscious or intellectual development, but simply that my body was making decisions for itself and yes, a unique language of movement was emerging from the confrontation of these two dance forms ». Cité in Annalisa PICcirillo, "Hybrid Bodies in Transit: the 'third language' of contemporary kathak », Anglistica, Napoli, Università degli Studi di Napoli « L'Orientale », Vol. 12, nº 2, 2008, p. 30.

41. Nous empruntons le terme d'« identité somatique " à Guy Cools qui revendique le fait que toute identité est toujours définie somatiquement - donc par le travail corporel -, autre que culturellement.

42. Ramsay BURT, "Contemporary dance and the performance of multicultural identities", Conference on research in dance, Copenhagen, The Ministry of Culture Committee on Sports Research, 8-9 janvier 2004, p. 1-13.

\section{AUTEUR}

\section{FEDERICA FRATAGNOLI}

Maîtresse de conférences en Danse à l'Université de Nice Sophia Antipolis, membre du « Centre Transdisci-plinaire d'Épistémologie de la Littérature et des Arts vivants » (CTEL) et membre associée du laboratoire « Musidanse » (Université Paris 8). Formée à la danse contemporaine et au bharata natyam, style de danse-théâtre du sud-est de l'Inde, elle a obtenu un doctorat en danse à l'Université de Paris 8, sous la direction d'I. Launay et J.M. Pradier. Entre théorie et pratique, sa recherche et son enseignement portent sur la lecture et l'analyse du geste dansé, ainsi que sur l'étude de langages chorégraphiques contemporains en lien avec la circulation de savoirs corporels indiens. Elle est membre de l'association des Chercheurs en danse et a fait partie de 2012 à 2017 du comité éditorial de la revue Recherches en danse. (http:// danse.revues.org/) 